\title{
Pessoas Idosas e Vulnerabilidade para Feridas Vasculogênicas: Marcadores para o Cuidado e o Envelhecimento Ativo
}

Elders and Vulnerability to Vascular Wounds: Markers for the Care and Active Ageing Ancianos y Vulnerabilidad para las Lesiones Vasculares: Marcadores para el
Cuidado y el Envejecimiento Activo Nathália Alvarenga-Martins', Helena Megumi Sonobe², Cristina Arreguy-Sena', Luciene Muniz Braga³, Luana Mendes de Souza ${ }^{4}$

\begin{abstract}
RESUMO
Pesquisa tipo convergente assistencial que objetivou analisar a vulnerabilidade de pessoas com 65 anos ou mais para ocorrência de feridas vasculogênicas a partir de parâmetros clínicos não invasivos, na busca por marcadores para subsidiar o cuidado e o processo de envelhecimento ativo e reunir evidências para estabelecer recomendações terapêuticas coerentes com as vulnerabilidades identificadas, com uniformização de terminologias para prescrição e avaliação de intervenções de enfermagem. Investigação realizada numa Unidade de Atenção Primária à Saúde do interior de Minas Gerais. Amostra por conglomerado. Foram atendidos os requisitos éticos e legais para pesquisa envolvendo seres humanos. Participaram 70 pessoas com mais de 65 anos de idade, residentes na área de abrangência de uma Unidade de Atenção Primária à Saúde, sendo identificado risco para úlceras vasculogênicas em $41,1 \%$ dos participantes. Foram propostas intervenções segundo Nursing Intervention Classification (NIC) e avaliação dos resultados segundo Nursing Outcome Classification (NOC). A magnitude da vulnerabilidade à ocorrência de doença arterial obstrutiva periférica alerta para uma lacuna na abordagem preventiva de lesões vasculogênicas, tendo em vista que não houve procura por serviços de saúde para este fim ou aproveitamento das oportunidades de consultas com outras finalidades para que intervenções preventivas fossem realizadas.
\end{abstract}

DESCRITORES: Estomaterapia. Enfermagem. Úlcera da Perna. Envelhecimento.

\begin{abstract}
Assistant convergence type which aimed to analyze the vulnerability of people aged 65 years or older to occur vasculogenic wounds from noninvasive clinical parameters in view of the search for markers to subsidize the care and the process of active aging, and gather evidence to outline treatment recommendations consistent with the identified vulnerabilities, using the unifying language to name them. Research conducted in Health Unit Primary in Minas Gerais. Cluster sample. Legal and ethical requirements for research involving human subjects were met. Participated in 70 people over 65 years of age living in the catchment area of a unit of Primary Health Care, identified some risk to vasculogenic ulcers in $41.1 \%$ of participants. Were proposed interventions seconds Nursing Intervention Classification (NIC) and evaluation of results according Nursing Outcomes Classification (NOC). The magnitude of vulnerability to the occurrence of peripheral arterial disease alert to a gap in preventative approach vasculogenic injuries, given that there was no demand for health services for this purpose nor use of opportunities of queries for other purposes for which interventions preventive were performed.
\end{abstract}

DESCRIPTORS: Stomatherapy. Estomaterapia. Nursing. Leg Ulcer. Aging.

${ }^{1}$ Faculdade de Enfermagem da Universidade Federal de Juiz de Fora (UFJF) - Juiz de Fora (MG), Brasil. Endereço para correspondência: Nathália Alvarenga-Martins - Rua Espírito Santo, 1286 - Centro - CEP: 36016-200 - Juiz de Fora (MG), Brasil - E-mail: nath.alvarenga.martins@gmail.com Escola de Enfermagem de Ribeirão Preto da Universidade de São Paulo (USP) - Ribeirão Preto (SP), Brasil.

${ }^{3}$ Faculdade de Enfermagem da Universidade Federal de Viçosa - Viçosa (MG), Brasil.

${ }^{4}$ Instituto de Previdência dos Servidores Militares de Minas Gerais - Juiz de Fora (MG), Brasil.

Artigo recebido em: 07/06/2015 - Aceito para publicação em: 19/07/2015 


\section{RESUMEN}

Investigación tipo convergente asistente que tuvo como objetivo analizar la vulnerabilidad de personas de 65 años o más para la ocurrencia de heridas vasculogénicas por medio de parámetros clínicos no invasivos, en la búsqueda de marcadores para subsidiar el cuidado y el proceso de envejecimiento activo y reunir evidencias para establecer las recomendaciones de tratamiento consistentes con las vulnerabilidades identificadas, utilizando terminologías uniformes para la prescripción y la evaluación de las intervenciones de enfermería. El estudio fue realizado en la Unidad Primaria de Salud en Minas Gerais. Muestreo por conglomerado. Se han cumplido los requisitos legales y éticos para la investigación en seres humanos. Participaron 70 personas de más de 65 años de edad, que vivían en la zona de captación de una Unidad de Atención Primaria de Salud, siendo identificado un riesgo para heridas vasculogénicos en el 41,1\% de los participantes. Fueron propuestas intervenciones, según la Clasificación de Intervenciones de Enfermería (NIC) y la evaluación de los resultados de acuerdo con la Clasificación de Resultados de Enfermería (NOC/CRE). La magnitud de la vulnerabilidad a la ocurrencia de la enfermedad arterial obstructiva periférica advierte para la necesidad del enfoque preventivo para lesiones vasculogénicas, siendo que no había ninguna demanda de servicios de salud para este propósito o uso de las oportunidades de consultas para otras finalidades para que se pudieran realizar intervenciones preventivas.

DESCRIPTORES: Estomaterapia. Enfermería. Úlcera de la Pierna. Envejecimento.

\section{INTRODUÇÃO}

No Brasil, define-se a entrada no processo do envelhecimento pela idade cronológica a partir dos 60 anos, enquanto, nos países desenvolvidos, a idade considerada é 65 anos. A população brasileira está estimada em 190.723.694 habitantes, sendo que a população idosa representa $11 \%$ da população total, $5 \%$ dela composta por homens e $6 \%$ por mulheres ${ }^{1}$.

Face as projeções da população com 60 anos de idade ou mais para os anos de 2025 e 2050, há conjunção de esforços dos profissionais de saúde, pessoas idosas e seus familiares no sentido de buscarem meios e recursos capazes de favorecer um processo de envelhecimento com redução das incapacidades funcionais ${ }^{2,3}$.

A Estomaterapia, como uma especialidade da enfermagem responsável pela assistência de pessoas com estomias, feridas e incontinências urinária e intestinal, tem importante papel no manejo do processo de envelhecimento ${ }^{4}$. Isso porque o envelhecimento representa significativo fator de risco para o desenvolvimento de algumas comorbidades, como as de origem vasculogênicas, que podem resultar na necessidade de tratamento de feridas crônicas 5 .

No âmbito das feridas vasculogênicas, o enfermeiro deve atuar na prevenção, detecção precoce de risco e recuperação. Esta atuação pode se dar de forma autônoma ou enquanto integrante de uma equipe multiprofissional, com intervenções que permeiam a assistência direta, a educação em saúde e o estímulo à pessoa idosa pela busca e manutenção de sua autonomia, possibilitando um envelhecimento ativo ${ }^{5-7}$.

O termo "envelhecimento ativo" está vinculado à concepção de saúde ampliada, o que equivale a retratar a preocupação com: a saúde e o bem-estar; a integração das pessoas em fase de envelhecimento dentro do ciclo de vida; a sua inserção no mercado de trabalho (in)formal; a sua participação e integração nos programas de formação continuada, a possibilidade e direito de acesso aos bens e às tecnologias disponíveis para promover a saúde e integração plena e maximizada aos bens disponibilizados pela sociedade ${ }^{8,9}$.

A prevenção de agravos e identificação precoce de vulnerabilidade para úlceras vasculogênicas é passível através de estratégias de baixo custo e proporcionam impacto sobre o processo de envelhecimento ativo ${ }^{10,11}$. Buscas de tecnologias sustentáveis, não invasivas e factíveis do ponto de vista da atenção primária são estratégias capazes de subsidiar a assistência de enfermagem junto à pessoa idosa com risco para úlceras vasculogênicas ${ }^{12}$.

Diante do exposto, este estudo justifica-se pela utilização de parâmetros clínicos não invasivos, constituindo uma abordagem de baixo custo, compatível com a prática laboral de enfermagem na atenção primária à saúde e capaz de identificar risco para ocorrência de úlceras vasculogênicas; identificação de risco para ocorrência de úlcera vasculogênicas, ou seja, de eventos intervenientes sobre a capacidade funcional e o envelhecimento saudável e ativo da pessoa com 60 anos de idade ou mais e a prevenção do surgimento de úlcera vasculogênica pela identificação de riscos para sua ocorrência e pela adoção de intervenções para redução destes riscos.

\section{OBJETIVO}

Analisar a vulnerabilidade de pessoas com 65 anos ou mais para ocorrência de feridas vasculogênicas a partir de parâmetros clínicos, na busca por marcadores para subsidiar o cuidado e o processo de envelhecimento ativo, e reunir evidências para estabelecer recomendações terapêuticas coerentes com as vulnerabilidades identificadas, com uniformização de terminologia para prescrição e avaliação de intervenções de enfermagem. 


\section{MÉTODO}

Pesquisa tipo convergente assistencial ${ }^{13,14}$ com população de seleção completa de cinco microáreas cadastradas em uma Unidade de Atenção Primária à Saúde (UAPS) de uma cidade do interior de Minas Gerais. A UAPS realizava a cobertura de 4.600 famílias e não possuía Estratégia de Saúde da Familia, funcionando segundo Modelo Tradicional.

Os participantes deste estudo foram recrutados a partir de visita domiciliar da equipe de saúde da referida UAPS, tendo sido necessários dois encontros; o primeiro para apresentação e agendamento de retorno, e o segundo para realização da coleta de dados propriamente dita e assinatura do Termo de Consentimento Livre e Esclarecido (TCLE). Foram critérios de elegibilidade: pessoas de ambos os gêneros, com 65 anos de idade ou mais, sem distinção de nível de escolaridade, racial ou étnica; usuários do Sistema Único de Saúde (SUS) na área de abrangência onde os dados foram coletados e que concordaram em participar como voluntários não remunerados.

Foram excluídos do estudo sete potenciais participantes que não atenderam aos critérios de inclusão, sendo dois porque não foram encontrados após três visitas domiciliares subsequentes; dois por mudança de endereço; um pelo fato de se encontrar internado durante o período de coleta de dados e dois por haverem se recusado a participar da pesquisa ou desejado interromper a participação ao longo de sua realização.

Utilizou-se instrumento de coleta de dados contendo:

1. caracterização sociodemográfica;

2. avaliação clínica dos Membros Inferiores (MMII) quanto à pigmentação, edema, integridade, queixa de dor em queimação, sensação de peso/cansaço em MMII, comorbidades e comportamentos de risco;

3. perfil de procura por atendimento de saúde e

4. aferição do Índice de Tornozelo-Braquial (ITB) para avaliação de Doença Arterial Obstrutiva Periférica (DAOP).

O questionário aplicado se compôs de avaliação clínica e questões fechadas. Os dados foram coletados de outubro de 2013 a abril de 2014.

Para a mensuração e cálculo do ITB, aferiram-se os índices pressóricos das artérias braquiais e pediosas, conforme VI Diretrizes Brasileiras de Hipertensão Arterial Sistêmica e protocolos ${ }^{15}$. Os usuários foram mantidos em repouso por cinco minutos e em decúbito dorsal, e a Pressão Arterial Sistólica (PAS) foi aferida nos quatro membros (artérias pediosas e braquiais direita e esquerda). O ITB foi calculado com base na maior PAS registrada nos Membros Superiores (MMSS) e MMII. O valor foi obtido mediante divisão da maior PAS em cada artéria dos MMII pela maior PAS obtida nos MMSS, conforme a fórmula para ITB. Os valores de referência foram: ITB anormal $<0,90$ e
$>1,30$; ITB normal de 0,91 a 1,2911,16. Cabe mencionar que, para elaborar a proposta de intervenções compatíveis com os possíveis problemas identificados, foram utilizadas as taxonomias Nursing Intervention Classification (NIC) e Nursing Outcome Classification (NOC).

Os dados foram consolidados em planilha do programa estatístico SPSS e tratados por análise descritiva e por testes de correlação.

O estudo atendeu todas as recomendações éticas e legais de pesquisa envolvendo seres humanos, em consonância com legislação brasileira, tendo sido o projeto aprovado em Comitê de Ética sob no 341.116. Pesquisa envolvendo riscos mínimos.

\section{RESULTADOS}

Participaram do estudo 70 pessoas com 65 anos de idade ou mais, sendo $43(61,4 \%)$ mulheres; idade média de 73,46 anos (variabilidade 65 a 92 anos e desvio padrão 6,26); 36 (51,4\%) eram casados e $34(48,6 \%)$ viviam sem companheiro(a).

Em relação à renda, $36(51,4 \%)$ recebiam um salário mínimo; ao grau de escolaridade, 24 (34,3\%) eram analfabetos, 64 (91,5\%) tinham até 8 anos de estudo, sendo que 3 (4,3\%) deles tinham variabilidade máxima de 11 anos de estudo. Dos participantes, 64 (91,4\%) usavam exclusivamente o SUS.

Quando perguntados sobre diagnóstico médico de Hipertensão Arterial Sistêmica (HAS) e Diabetes Mellitus (DM), $52(74,3 \%)$ e 23 (32,9\%), respectivamente, responderam afirmativamente. Quanto aos comportamentos de riscos, 9 (12,9\%) disseram fazer uso de bebidas alcoólicas e 14 (20\%) usar tabaco.

Ao realizar o exame clínico dos MMII nos participantes, foi verificado hiperpigmentação em 19 (27,1\%), varizes em $36(51,4 \%)$, edema em 17 (24,3\%), úlceras vasculogênicas em 5 (7,1\%). Quando questionados em relação à sensação de peso/cansaço em MMII e dor em queimação, $29(41,4 \%)$ e $10(14,3 \%)$, respectivamente, responderam afirmativamente.

A Tabela 1 apresenta os resultados dos índices tornozelo-braquial e equivalência para gravidade da Doença Obstrutiva Arterial Periférica.

Foram realizadas correlações entre as variáveis clínicas observadas e o ITB, demonstrados na Tabela 2.

Foi realizado, ainda, Teste $t$ de Student para amostra simples para variáveis intervenientes, manifestações, morbidades e comportamentos de risco (Tabela 3).

Com base nas recomendações consensualizadas internacionalmente, foi possível elaborar o Quadro 1, no qual estão consolidadas ações de caráter preventivo para o desenvolvimento de úlceras vasculogênicas. 
Tabela 1. Índices tornozelo-braquial, equivalência para gravidade da Doença Obstrutiva Arterial Periférica. Jan/2015.

\begin{tabular}{|c|c|c|c|}
\hline $\begin{array}{l}\text { Valores } \\
\text { ITB }\end{array}$ & $\begin{array}{c}\text { Gravidade } \\
\text { DAOP }\end{array}$ & $\mathrm{n}$ & $\%$ \\
\hline$\leq 0,41$ & DAOP Grave & 1 & 1,4 \\
\hline $0,41-0,7$ & DAOP Moderada & 9 & 12,9 \\
\hline $0,7-10,9$ & DAOP Leve & 12 & 17,1 \\
\hline $0,9-1,3$ & Normal & 41 & 58,6 \\
\hline$\geq 1,3$ & $\begin{array}{c}\text { Doença } \\
\text { aterosclerótica } \\
\text { grave }\end{array}$ & 7 & 10,0 \\
\hline Total & & 70 & 100,0 \\
\hline
\end{tabular}

ITB: Índice de Tornozelo-Braquial; DAOP: Doença Obstrutiva Arterial Periférica

Tabela 2. Correlações entre as variáveis clínicas observadas e o Índice de Tornozelo-Braquial. Juiz de Fora (MG); Jan/2015.

\begin{tabular}{lcc}
\hline Variável & ITB & Valor $\mathbf{p}$ \\
\hline Idade & 0,500 & 0,680 \\
\hline Hiperpigmentação & $-0,286^{*}$ & 0,160 \\
\hline Varizes & $-0,303^{* \star}$ & 0,011 \\
\hline Edema & $-0,549^{* \star}$ & 0,000 \\
\hline
\end{tabular}

ITB: Índice de Tornozelo-Braquial; *Correlação de Pearson com nível de significância 0,05; **Correlação de Pearson com nível de significância 0,01.

\section{DISCUSSÃO}

A população pesquisada possui idade média coincidente com a expectativa de vida da população brasileira, e o processo de feminização do envelhecimento, que ocorre nacional e internacionalmente, também pode ser confirmado entre os participantes ${ }^{17}$.

A média de analfabetismo entre os participantes foi ligeiramente inferior à média nacional; porém, a maior parte deles tinha no máximo oito anos de estudo ${ }^{2}$. A renda familiar foi considerada baixa, sendo a maioria de um salário mínino.

O conjunto das variáveis baixa renda, gênero feminino e baixo nível de instrução encontradas na investigação é uma constante em estudos cuja população apresentava úlceras de origem vascular ${ }^{18,19}$. Este conjunto de variáveis pode ser preditor para comportamentos que favoreçam o desenvolvimento de afecções vasculares periféricas ${ }^{20} \mathrm{e}$, quando aliado à idade, tem-se um elenco de características que devem alertar os profissionais de saúde para o risco de desenvolvimento de
Tabela 3. Teste $t$ de Student para uma amostra simples, tendo como variáveis intervenientes as manifestações, morbidades e comportamentos de risco. Juiz de Fora (MG); Jan/2015.

\begin{tabular}{|c|c|c|c|}
\hline Variáveis & $\mathrm{t}$ & $\begin{array}{c}\text { Valor } \\
\mathrm{p}\end{array}$ & $\begin{array}{l}\text { Diferença } \\
\text { média }\end{array}$ \\
\hline ITB & 12,08 & 0,00 & 1,87 \\
\hline $\begin{array}{l}\text { Comportamento } \\
\text { para uso de álcool }\end{array}$ & 33,01 & 0,00 & 1,81 \\
\hline $\begin{array}{l}\text { Comportamento } \\
\text { para uso de tabaco }\end{array}$ & 37,38 & 0,00 & 1,80 \\
\hline Dor em queimação & 44,08 & 0,00 & 1,85 \\
\hline Edema & 34,03 & 0,00 & 1,75 \\
\hline Hiperpigmentação & 32,28 & 0,00 & 1,72 \\
\hline $\begin{array}{l}\text { Lesões } \\
\text { vasculogênicas }\end{array}$ & 62,20 & 0,00 & 1,92 \\
\hline $\begin{array}{l}\text { Sensação de } \\
\text { peso/cansaço }\end{array}$ & 26,74 & 0,00 & 1,58 \\
\hline Varizes & 24,69 & 0,00 & 1,48 \\
\hline
\end{tabular}

ITB: Índice de Tornozelo-Braquial

úlceras vasculogênicas, cabendo intervenção preventiva, de competência de enfermeiros generalistas, envolvendo tecnologia de baixo custo ${ }^{18,19}$.

O serviço público nacional tem apresentado carência de acessibilidade às especialidades de angiologia e estomaterapia, fazendo com que as avaliações e abordagens de caráter preventivo de úlceras vasculogênicas ocorram quando as lesões já estão instaladas, ou seja, tardiamente ${ }^{20}$.

O fato de $91,4 \%$ dos participantes utilizarem exclusivamente o SUS como serviço para assistência à saúde constituiu um marcador de vulnerabilidade para lesões de MMII, na medida em que esta questão ainda não havia sido investigada entre eles e nem ocorreu tratamento preventivo nos serviços em que foram atendidos ${ }^{20}$.

A investigação de diagnóstico prévio e/ou sinais e sintomas de DM se deu por esta ser uma doença crônica e multifatorial que, devido a macro e microangiopatias e/ou neuropatias, leva a complicações que podem afetar inúmeros órgãos, dentre eles, a pele. Quando o DM está associado à HAS, tem-se um aumento dos riscos para desenvolvimento de úlceras de perna; portanto, estas doenças requerem identificação e tratamento previsto em protocolo, visando à prevenção de complicações do sistema vascular e da pele ${ }^{21}$.

Alguns comportamentos são considerados de risco para o desenvolvimento de úlceras vasculogênicas, entre eles consumir tabaco e bebida alcoólica ${ }^{5,10}$. Pensando em uma assistência de enfermagem individualizada, fazem-se 
Quadro 1. Protocolo baseado nas evidências de risco para desenvolvimento de úlceras vasculogênicas, identificadas neste estudo. Juiz de Fora (MG); Jan/2015.

\begin{tabular}{|c|c|c|}
\hline $\begin{array}{l}\text { Fator de } \\
\text { vulnerabilidade }\end{array}$ & Intervenções (NIC) & Avaliação dos Resultados (NOC) \\
\hline Hiperpigmentação & $\begin{array}{l}\text { Controle de medicamentos } \\
\text { (D2, CH); Controle da perfusão } \\
\text { tissular (D2, CN); Controle de } \\
\text { pele/feridas (D2, CL);Controle } \\
\text { de riscos (D4, CV). }\end{array}$ & $\begin{array}{l}\text { Integridade tissular: pele e mucosas (D2, CL); } \\
\text { Perfusão tissular: periférica (D2, CE); Satisfação } \\
\text { do cliente: cuidado físico (D2, CE). }\end{array}$ \\
\hline Varizes & $\begin{array}{l}\text { Controle da perfusão tissular } \\
(\mathrm{D} 2, \mathrm{CN}) \text {. }\end{array}$ & $\begin{array}{l}\text { Estado circulatório (D2, CE); Integridade tissular } \\
\text { pele e mucosas (D2, CL); Satisfação do cliente } \\
(\mathrm{D} 5, \mathrm{CE}) \text {. }\end{array}$ \\
\hline Edema & $\begin{array}{l}\text { Controle da imobilidade (D1, } \\
\text { CC); Apoio nutricional (D1,CD); } \\
\text { Controle da perfusão tissular } \\
\text { (D2, CN). }\end{array}$ & $\begin{array}{l}\text { Mobilidade (D1,C C); Estado nutricional (D2, CK); } \\
\text { Estado circulatório (D2, CE); Integridade tissular: } \\
\text { pele e mucosas (D2, CL); Satisfação do cliente } \\
\text { assistência funcional (D5, CE). }\end{array}$ \\
\hline Dor & $\begin{array}{l}\text { Promoção do conforto } \\
\text { físico (D1, CE); Controle de } \\
\text { medicamentos (D2, CH); } \\
\text { Controle da atividade e do } \\
\text { exercício (D1, CA). }\end{array}$ & $\begin{array}{l}\text { Estado do sintoma (D5, CU); Perfusão tissular: } \\
\text { periférica (D2, CE); Controle da dor (D4, } \\
\text { CQ); Controle de sintomas (D4, CQ);Nível de } \\
\text { desconforto (D5, CV);Dor: resposta psicológica } \\
\text { adversa (D5, CV);Satisfação do cliente (D5, CE). }\end{array}$ \\
\hline HAS/DM & $\begin{array}{l}\text { Controle de medicamentos (D2, } \\
\mathrm{CH}) \text {; Apoio nutricional (D1, CD). }\end{array}$ & $\begin{array}{l}\text { Estado circulatório (D2, CE); Nível de glicemia } \\
\text { (D2, CA); Comportamento de adesão (D4, CQ); } \\
\text { Comportamento de aceitação: dieta prescrita } \\
\text { (D4, CQ): Comportamento de aceitação: } \\
\text { medicação prescrita (D4, CQ); Autocontrole } \\
\text { do Diabetes (D4, CQ); Comportamento de } \\
\text { Promoção da saúde (D4, CQ); Controle de } \\
\text { riscos: saúde cardiovascular (D4, CT); Satisfação } \\
\text { do cliente: Ensino (D5, CE). }\end{array}$ \\
\hline Tabaco & $\begin{array}{l}\text { Terapia comportamental (D3, } \\
\text { CO); Terapia cognitiva (D3, CP). }\end{array}$ & $\begin{array}{l}\text { Comportamento de Promoção da saúde (D4, CQ); } \\
\text { Comportamento de cessação do tabaco (D4, CQ). }\end{array}$ \\
\hline Álcool & $\begin{array}{l}\text { Terapia cognitiva (D3, CP); } \\
\text { Assistência no enfrentamento } \\
\text { (D3, CR); Educação do paciente } \\
\text { (D3, CS). }\end{array}$ & $\begin{array}{l}\text { Comportamento de suspensão do abuso de } \\
\text { álcool (D4, CQ); Comportamento de Promoção } \\
\text { da saúde (D4, CQ). }\end{array}$ \\
\hline $\begin{array}{l}\text { Abordagem do } \\
\text { indivíduo com ITB } \\
\text { alterado }\end{array}$ & $\begin{array}{l}\text { Assistência no enfrentamento } \\
\text { (D3, CR); Educação do } \\
\text { paciente (D3, CS); Controle das } \\
\text { informações(D6, CB); Mediação } \\
\text { do sistema de saúde (D6, CY); }\end{array}$ & $\begin{array}{l}\text { Enfrentamento (D3, CN); Função sensorial } \\
\text { cutânea (D2, CY); Estado circulatório (D2, CE); } \\
\text { Perfusão tissular: periférica (D2, CE); Qualidade } \\
\text { de vida (D5, CU); Comportamento de adesão } \\
\text { (D4, CQ); Comportamento de Promoção da } \\
\text { saúde (D4, CQ); Comportamento de busca da } \\
\text { saúde (D4, CQ); Participação sobre cuidados } \\
\text { de saúde (D4, CQ); Orientação para saúde (D4, } \\
\text { CR); Conhecimento: promoção da saúde (D4, } \\
\text { CS); Conhecimento: recursos de saúde (D4, CS); } \\
\text { Controle de riscos (D4, CT); Estado de conforto } \\
\text { (D5, CU); Satisfação do cliente (D5, CE). Controle } \\
\text { de riscos comunitários: doença crônica (D7, CC). }\end{array}$ \\
\hline
\end{tabular}

NIC: Nursing Intervention Classification; NOC: Nursing Outcome Classification; D2: Domínio 2; CH: Classe H; CN: Classe N; CL: Classe L; D4: Domínio 4; CV: Classe V; CE: Classe E; D1: Domínio 1; CC: Classe C; CD: Classe D; CK: Classe K; D5: Domínio 5; CA: Classe A; CU: Classe U; CQ: Classe Q; CT: Classe T; D3: Domínio 3; CO: Classe O; CP: Classe P; CR: Classe R; CS: Classe S; D6: Domínio 6; CB: Classe B; CY: Classe Y; D7: Domínio 7. 
necessárias intervenções para auxiliar a pessoa que fuma ou ingere bebida alcoólica a abandonar esses hábitos 5 . Para os casos de dependência severa do álcool e/ou tabaco, existem inúmeras abordagens e tratamentos que incluem intervenções farmacológicas e psicocomportamentais ${ }^{22}$. O hábito de fumar e de beber está intimamente ligado ao surgimento de DM e HAS e, por isso, essas doenças merecem ser consideradas na prevenção de úlceras vasculogênicas ${ }^{10}$.

Um estudo realizado em um hospital público na cidade do Rio de Janeiro (RJ) com pessoas com idade $\geq 50$ anos apresentou prevalência do ITB alterado por influência do tabagismo, HAS e $\mathrm{DM}^{10}$, fatores que também comprometem o processo de envelhecimento ativo?.

Os sinais clínicos (varizes, edemas, hiperpigmentação, queixa de dor em queimação e sensação de peso/cansaço) avaliados durante o exame dos MMII estão associados à situação de comprometimento do sistema vascular periférico ${ }^{20}$ A presença de varizes pode ser explicada pela insuficiência valvar; o edema está intimamente ligado à permeabilidade do vaso e migração do líquido seroso para o interstício, e a hiperpigmentação está associada à deposição de hemossiderina pela degradação das hemácias em decorrência do retorno venoso comprometido ${ }^{20,23}$.

O relato afirmativo de dor em queimação e sensação de peso/cansaço deve ser visto como sinal de alerta em decorrência da ineficácia da perfusão sanguínea, que pode evoluir para necrose tecidual e manifestar-se em ferida ${ }^{16,24}$ A sensação de peso/cansaço pode estar intimamente relacionada ao edema ${ }^{24}$.

Apesar de o foco da presente pesquisa estar na avaliação precoce de riscos para ocorrência de úlcera vasculogênica, esta foi investigada também entre aqueles participantes que já possuíam uma lesão, em decorrência da possibilidade de recidiva ou do surgimento de mais uma úlcera no mesmo membro ou em membro diferente ${ }^{7,20}$.

$\mathrm{Na}$ Tabela 1, apesar de 58,6\% dos participantes apresentarem índice tornozelo-braquial na faixa de normalidade, $31,4 \%$ deles apresentam risco de DAOP nos níveis leve e moderado. Cabe acrescentar que $10 \%$ deles estão expostos ao risco de doença aterosclerótica grave. Torna-se fundamental que intervenções preventivas sejam pensadas quando se almeja alcançar as metas propostas na política do envelhecimento ativo ${ }^{9,11,16}$. Caso estas intervenções não sejam realizadas, essa população estará vulnerável a complicações, o que impacta a qualidade de vida das pessoas ido$\mathrm{sas}^{18}$, o financiamento do SUS ${ }^{12}$ e o surgimento de síndromes geriátricas (alteração na mobilidade e na independência) ${ }^{8}$.

As correlações entre ITB e as características de hiperpigmentação, varizes e edema apresentaram valores com significância estatística (Tabela 2) que podem estar atrelados ao grau de gravidade da $\mathrm{DAOP}^{11}$. Essas características, como já citado, vêm em decorrência da circulação vascular comprometida e foram encontradas em outras pesquisas ${ }^{10,19,20,24}$.
Tais evidências reforçam a necessidade de uma abordagem sistêmica e multidisciplinar na prevenção de complicações em decorrência do comprometimento vascular, para assegurar capacidade funcional, bem-estar e qualidade de vida para a pessoa idosa com risco para desenvolvimento de úlcera vasculogênica ${ }^{18}$.

$\mathrm{Na}$ Tabela 3, resultados de teste $t$ de Student para amostra simples mostraram significância estatística para as variáveis ITB, consumo de álcool e tabaco e sinais e sintomas como: dor em queimação, hiperpigmentação, lesões vasculogênicas, sensação de peso/cansaço e varizes, corroborando evidências apontadas nacional e internacionalmente ${ }^{11,20,24}$.

Com base nas recomendações consensualizadas internacionalmente, foi possível elaborar o Quadro 1, no qual estão consolidadas ações de caráter preventivo para o desenvolvimento de úlceras vasculogênicas segundo taxonomias da Classificação das Intervenções de Enfermagem (NIC) ${ }^{25} \mathrm{e}$ da Classificação dos Resultados de Enfermagem $(\mathrm{NOC})^{26}$.

Tendo como base as taxonomias da $\mathrm{NIC}^{25} \mathrm{e} \mathrm{da} \mathrm{NOC}^{26}$ e a partir dos fatores de vulnerabilidade encontrados na população estudada, o Quadro 1 consolidou intervenções e os critérios passíveis de avaliar os resultados esperados.

Para o fator Hiperpigmentação, selecionou-se as seguintes intervenções ${ }^{25}$ - Controle de medicamentos: administração tópica (hidratação); Controle da perfusão tissular: Cuidados circulatórios: insuficiência arterial; Cuidados circulatórios: insuficiência venosa. Controle de pele/feridas: Monitoração das exterminadas inferiores; Controle da pressão; Cuidados da pele: tratamentos tópicos (Hidratação); Supervisão da pele; Controle de riscos: Avaliação da saúde; Identificação de risco; Facilitação do autocuidado: assistência no autocuidado; assistência no autocuidado: transferência.

Os critérios para avaliação dos resultados para o fator Hiperpigmentação, foram - Integridade tissular: pele e mucosas: Textura; Perfusão Tissular; Integridade da pele; Hidratação; Pigmentação anormal; Pele que descasca; Descamação da pele: Eritema; Perfusão tissular: periférica: Rubor; Satisfação do cliente: cuidado físico: Rotina de cuidados da pele mantida; Cuidados especiais de pele seguidos ${ }^{26}$.

Para o fator de vulnerabilidade Varizes, as intervenções previstas foram - Controle da perfusão tissular: cuidados circulatórios: equipamentos de suporte circulatório mecânico (meia elástica); Cuidados circulatórios: insuficiência venosa ${ }^{25}$. E para avaliação dos resultados - Estado circulatório: Preenchimento capilar; Claudicação intermitente; Integridade tissular pele e mucosas: Perfusão tissular; Satisfação do cliente: Cuidados para prevenir danos ou lesões; Rotina de cuidados da pele mantida; Cuidados especiais de pele seguidos ${ }^{26}$.

O fator de vulnerabilidade Edema teve como intervenções selecionadas- Controle da imobilidade: Posicionamento; Cuidados com repouso no leito; Apoio nutricional: Planejamento da dieta; Aconselhamento nutricional; Ensino: 
dieta prescrita; Controle da perfusão tissular: Cuidados circulatórios: equipamentos de suporte circulatório mecânico (meia elástica ${ }^{25}$.E para avaliação dos resultados - Mobilidade: Marcha; Movimentos realizados com facilidade; Estado nutricional: Ingestão de sódio; Estado circulatório: Preenchimento capilar; Edema periférico; Claudicação intermitente; Edema depressível; Integridade tissular: pele e mucosas: Perfusão tissular; Integridade da pele, Endurecimento; Satisfação do cliente assistência funcional: Inclusão no planejamento para melhora da mobilidade e do cuidado; Rotina de cuidados da pele mantida; Cuidados especiais de pele seguidos; Assistência na locomoção; Assistência nas transferências Controle ${ }^{26}$.

Em relação ao fator de vulnerabilidade Dor, foram selecionadas as seguintes intervenções - Promoção do conforto físico: Controle da dor; Controle de medicamentos: Administração de analgésicos; Controle da atividade e do exercício: Terapia com exercício: deambulação; Ensino: atividade/exercícios prescritos ${ }^{25}$.

E para avaliação dos resultados para o fator Dor Estado do sintoma: Estado de conforto físico; Controle de sintomas; Perfusão tissular: periférica: Dor localizada em extremidades; Controle da dor: Reconhecimento do início da dor: Descrição dos fatores causadores; Uso de medidas preventivas; Uso de medidas de alívio não analgésico; Uso de analgésicos conforme recomendação; Relato de dor controlada; Controle de sintomas: Uso de medidas de alívio dos sintomas; Nível de desconforto: Dor; Mobilidade física prejudicada; Sono interrompido; Dor: resposta psicológica adversa: Sofrimento decorrente da dor; Gravidade dos sintomas: Intensidade dos sintomas; Frequência dos sintomas; Persistência dos sintomas; Mobilidade física prejudicada; Satisfação do cliente: Alívio dos sintomas da doença; Cuidados para o controle da dor; Encaminhamentos a profissionais de saúde para manejo da dor, conforme a necessidade ${ }^{26}$.

Os fatores de vulnerabilidade HAS e DM foram agrupados e as intervenções previstas foram - Controle de medicamentos: Administração de medicamentos; Apoio nutricional: planejamento da dieta; Aconselhamento nutricional; Ensino: dieta prescrita ${ }^{25}$. E os resultados esperados - Estado circulatório: Pressão arterial sistólica; Pressão arterial diastólica; Estado nutricional: Ingestão de sódio; Ingestão de carboidrato; Nível de glicemia: Glicose do sangue; Comportamento de adesão: Realização de automonitoração do estado de saúde; Comportamento de aceitação: dieta prescrita: Ingestão de alimentos coerentes com a dieta prescrita; Comportamento de aceitação: medicação prescrita: Mantém uma lista de todos os medicamentos com dose e frequência; Obtém medicação necessária; Informa ao profissional de saúde de todos os medicamentos tomados; Autocontrole do Diabetes: Participação no processo decisório sobre cuidados de saúde; Uso de medidas preventivas para reduzir o risco de complicações; Comportamento de Promoção da saúde: Atendimento a uma dieta saudável;
Controle de riscos: saúde cardiovascular: Monitoração da pressão arterial; Atendimento à dieta recomendada; Satisfação do cliente: Ensino: Explicação das razões do tratamento; Explicação das responsabilidades pelo autocuidado no tratamento ${ }^{26}$.

Para o fator de vulnerabilidade Tabaco as intervenções selecionadas foram - Terapia comportamental: assistência para parar de fumar; Terapia cognitiva: facilitação da aprendizagem; melhora da disposição para aprender ${ }^{25}$. E para avaliação dos resultados - Comportamento de Promoção da saúde: Ato de evitar mau uso do tabaco; Comportamento de cessação do tabaco: Expressão do desejo de parar de fumar; Identificação dos benefícios de parar de fumar; Identificação das consequências negativas do uso do tabaco; Obtenção de assistência de profissional de saúde; Adaptação do estilo de vida para promover a eliminação do tabaco; Eliminação do uso do tabaco ${ }^{26}$.

Para o fator de vulnerabilidade Álcool selecionou-se as seguintes intervenções - Terapia cognitiva: facilitação da aprendizagem; melhora da disposição para aprender; Assistência no enfrentamento: melhora do enfrentamento; aumento da segurança; Educação do paciente: Educação à saúde ${ }^{25}$. Para avaliação dos resultados - Comportamento de suspensão do abuso de álcool: Expressa desejo de parar de usar álcool; Identifica benefícios da eliminação do uso de álcool; Identifica consequências negativas do uso de álcool; Desenvolve estratégias eficientes para eliminar o uso de álcool; Obtém assistência de profissional de saúde; Comportamento de Promoção da saúde: Ato de evitar mau uso do álcool ${ }^{26}$.

Para abordagem do indivíduo com ITB alterado, definiram-se as seguintes intervenções- Assistência no enfrentamento: melhora do enfrentamento; aumento da segurança; fortalecimento da autocompetência; Educação do paciente: Educação à saúde; Facilitação da aprendizagem; Melhora da disposição para aprender; Ensino processo da doença; Ensino: atividade/exercício prescrito; Controle das informações: encaminhamento (referência/contra referência); Mediação do sistema de saúde: Orientação quanto ao sistema de saúde; Facilitação da visita (visita domiciliar) ${ }^{25}$.

$\mathrm{E}$ como avaliação dos resultados diante alteração do ITB - Enfrentamento: Adaptação a mudanças no estilo de vida; Uso de estratégias eficientes de enfrentamento; Função sensorial cutânea: Formigamento; Estado circulatório: Parestesia: Perfusão tissular: periférica: Preenchimento capilar nos dedos dos pés; Qualidade de vida: Capacidade de enfrentamento; Comportamento de adesão: Realização de automonitoração do estado de saúde; Comportamento de Promoção da saúde: Uso de comportamentos para evitar riscos; Monitoração do comportamento pessoal com relação a riscos; Atendimento a uma dieta saudável; Comportamento de busca da saúde: Realização de autoavaliação; Desempenho de comportamento de saúde prescrito; Participação sobre 
cuidados de saúde: Mostra autodirecionamento nas tomadas de decisões; Negociação das preferências de cuidado; Orientação para saúde: Foco na prevenção de doenças; Foco no bem-estar geral; Conhecimento: promoção da saúde: Comportamentos que promovem a saúde; Conhecimento: recursos de saúde: Importância dos cuidados de acompanhamento; Estratégias de acesso a serviços de cuidados de saúde; Controle de riscos: Monitoração dos fatores de risco; Monitoração de fatores de risco ao comportamento pessoal; Desenvolvimento de estratégias eficientes de controle de riscos; Modificação do estilo de vida para reduzir os riscos; Uso dos serviços de cuidados de saúde coerentes com as necessidades; Reconhecimento de mudanças no estado de saúde; Monitoração de mudanças no estado de saúde; Estado de conforto: Bem-estar físico; Satisfação do cliente: Acesso à equipe de enfermagem; Acesso a suprimentos e equipamentos necessários aos cuidados; Encaminhamentos a profissionais de saúde para manejo da dor, conforme a necessidade; Informações dadas sobre riscos e as complicações do tratamento; Controle de riscos comunitários: doença crônica: Disponibilidade de programas preventivos de avaliaçãa ${ }^{26}$.

O conteúdo do Quadro 1 retrata ações compatíveis com a atuação do enfermeiro generalista ou enfermeiro estomaterapeuta na prevenção de úlceras vasculogênicas, que podem reduzir gastos públicos, impactar favoravelmente sobre a qualidade de vida da pessoa idosa, favorecer a manutenção da capacidade funcional e contribuir com práticas que fortaleçam os pilares do envelhecimento ativo ${ }^{9,18}$.

Diante do exposto, sugere-se a validação do conteúdo do Quadro 1 na realidade investigada e em outras de diferentes perfis.

\section{CONCLUSÃO}

A magnitude da vulnerabilidade à ocorrência de DAOP entre pessoas com idade $\geq 65$ anos a partir de parâmetros clínicos alerta para uma lacuna na abordagem preventiva de lesões vasculogênicas, tendo em vista que não houve procura dos serviços de saúde para este fim ou aproveitamento das oportunidades do momento das consultas para realização de abordagens de caráter preventivo.

Estes resultados apontam para um espaço passível de ser ocupado pelo enfermeiro generalista e pelo estomaterapeuta com vista à realização de intervenções educativas para o autocuidado das pessoas com idade $\geq 65$ anos para identificar se elas possuem ou não risco para ocorrência de feridas vasculogênicas.

\section{REFERÊNCIAS}

1. Nicodemo D, Godoi M. Juventude dos anos 60-70 e envelhecimento: estudo de casos sobre feminização e direitos de mulheres idosas. Revista Ciência em Extensão. 2010;6(1):40-53.

2. Instituto Brasileiro de Geografia e Estatística (IBGE). Síntese de Indicadores Sociais: Uma análise das Condições de Vida da População Brasileira. Rio de Janeiro: Estudos e Pesquisas Informações Demográfica e Socioeconômica número 29. 2014 [Internet]. [citado em 12 out 2014]. Disponível em: $\mathrm{ftp}: / /$ ftp.ibge.gov.br/Indicadores_Sociais/Sintese_de_ Indicadores_Sociais_2014/SIS_2014.pdf

3. Both JE, Beuter M, Perrando MS, Silva MS, Bruinsma JL, Rocha LS. Tendências na construção do conhecimento em enfermagem: idoso e autocuidado. Revista Brasileira de Ciências da Saúde. 2014;12(39):44-52.

4. Yamada BA, Ferrola EC, Azevedo GR, Blanes L, Rogenski NM, Santos VLCG. Competências do enfermeiro estomaterapeuta Ti SOBEST ou do enfermeiro estomaterapeuta. Rev Estima. 2008;6(1):33-43.

5. Medeiros ABA, Andriola IC, Fernandes MICD, Silva FBBL, Sá JD, Lira ALBC. Perfil socioeconômico de pessoas com úlcera venosa: aspectos relevantes para a enfermagem. Revista de Enfermagem UFPE. 2013;7(8):5220-4.

6. Caldas CP, Veras RP, Motta LB, Lima KC, Kisse CBS, Trocado CVM, et. al. Rastreamento do risco de perda funcional: uma estratégia fundamental para organização da Rede de Atenção ao Idoso. Ciên Saúde Colet. 2013;18(2):3495-506.
7. Silva DC, Durgante VL, Rizzatti SJS, Santos VC, Budó MLD, Farão EMD. Cuidado de enfermagem aos usuários com úlceras venosas. Revista Contexto \& Saúde. 2013;11(20):851-4.

8. Moraes EN. Atenção à saúde do idoso: aspectos conceituais. Brasília-DF: Organização Pan-Americana de Saúde. 2012. p. 98.

9. World Health Organization. Envelhecimento Ativo: uma política de saúde; tradução Seuzana Gontijo. Brasília: Organização Pan-Americana de Saúde. 2005; 60p.

10. Panico MDB, Spichler ES, Neves MF, Pinto LW, Spichler D. Prevalência e fatores de risco da doença arterial periférica sintomática e assintomática em hospital terciário. J Vasc Bras. 2009;8:125-32.

11. Maggi DL, Quadros LRDP, de Oliveira Azzolin K, Goldmeier S. Índice tornozelo-braquial: estratégia de enfermeiras na identificação dos fatores de risco para doença cardiovascular. Rev Esc Enferm USP. 2014;48(2):223-7.

12. Santos ICRV, Oliveira Souza MA, Andrade LNV, Lopes MP, Barros e Silva MFA, Santiago RT. Caracterização do atendimento de pacientes com feridas na Atenção Primária. Rev Rene. 2014;15(4):613-20

13. Paim L, Trentini M, Madureira VSF, Stamm M. Pesquisa convergente assistencial e sua aplicação em cenários da enfermagem. Cogitare Enferm. 2008;13(3):380-6.

14. Krempser P, Arreguy-Sena C, Barbosa APS. Características definidoras de trauma vascular periférico em urgência e emergência: ocorrência e tipos. Esc Anna Nery. 2013;17(1):24-30. 
15. Sociedade Brasileira de Cardiologia, Sociedade Brasielira de Hipertensão, Sociedade Brasileira de Nefrologia. VI Diretrizes Brasileira de Hipertensão. Arq Bras Cardiol. 2010;95(1supl.1):1-51.

16. Torres J, Machado AG, Lopes EGSL, Gentile PC, Vieira AC, Soares LG, et al. Prevalência de Alterações do Índice Tornozelo-Braço em Indivíduos Portadores Assintomáticos de Doença Arterial Obstrutiva Periférica. Rev Bras Cardiol. 2012;25(2):87-93.

17. Mello MM. O envelhecimento da população brasileira: intensidade, feminização e dependência. Rev Bras Estudo População. 1998;15(1):79-94.

18. Edwards H, Courtney M, Finlayson K, Shuter P, Lindsay E. A randomised controlled trial of a community nursing intervention: improved quality of life and healing for clients with chronic leg ulcers. J Clin Nurs. 2009;18(11):1541-9.

19. Torres GV, Costa IKF, Dantas DV, Dias T, Nunes JP, Deodato OON, et al. Idosos com úlceras venosas atendidos nos níveis primário e terciário: caracterização sociodemográfica, de saúde e assistência. Rev Enferm UFPE. 2009;3(4):222-30.

20. Malaquias SG, Bachion MM, Sant'Ana SMSC, Dallarmi CCB, Junior RdSL, Ferreira PS. Pessoas com úlceras vasculogênicas em atendimento ambulatorial de enfermagem: estudo das variáveis clínicas e sociodemográficas. Rev Esc Enferm USP. 2012;46(2):302-10.
21. Minatel DG, Enwemeka CS, França SC, Frade MAC. Fototerapia (LEDs 660/890nm) no tratamento de úlceras de perna em pacientes diabéticos: estudo de caso. An Bras Dermatol. 2009;84(3):279-83.

22. Senger AEV, Ely LS, Gandolfi T, Schneider RH, Gomes I, De Carli GA. Alcoolismo e tabagismo em idosos: relação com ingestão alimentar e aspectos socioeconômicos. Rev Bras Geriatr Gerontol. 2011;14(Supl 4):713-9.

23. Seidel AC, Mangolim AS, Rossetti LP, Gomes JR, Miranda Jr F. Prevalência de insuficiência venosa superficial dos membros inferiores em pacientes obesos e não obesos. J Vasc Bras. 2011;10(2):124-30

24 Salvetti MG, Costa IKF, Dantas DV, Freitas CCSd, Vasconcelos QLDAQ, Torres GV. Prevalência de dor e fatores associados em pacientes com úlcera venosa. Rev Dor. 2014;1 5(1):17-20.

25. Bulechek G, Butcher H, Dochterman J. NIC: Classificação da Intervenções de Enfermagem. $5^{\mathrm{a}}$ ed. Rio de Janeiro: Elsevier; 2010 944p.

26. Moorhead S, Johnson M, Maas ML, Swanson E. NOC: Classificação dos Resultados de Enfermagem. $4^{\text {a }}$ ed. Rio de Janeiro: Elsevier; 2010. 936 p. 